\title{
Regulation of homeobox A10 expression in the primate endometrium by progesterone and embryonic stimuli
}

\author{
G B Godbole, D N Modi and C P Puri \\ National Institute for Research in Reproductive Health, Indian Council of Medical Research, JM Street, Parel, Mumbai \\ 400012, India
}

Requests for offprints should be addressed to D N Modi; Email: deepaknmodi@yahoo.com, modidn@icmr.org.in

\begin{abstract}
Homeobox A10 (HOXA10), a member of abdominal B subclass of homeobox genes, is responsible for uterine homeosis during development. Intriguingly, in the adult murine uterus, HOXA10 has been demonstrated to play important roles in receptivity, embryo implantation, and decidualization. However, the roles of HOXA10 in the primate endometrium are not known. To gain insights into the roles of HOXA10 in the primate endometrium, its expression was studied in the endometria of bonnet monkey (Macaca radiata) in the receptive phase and also in the endometria of monkeys treated with antiprogestin onapristone (ZK98.299) or in conception cycle where the presence of preimplantation stage blastocyst was verified. In addition, the mRNA expression of HOXA11 and insulin-like growth factor-binding protein 1 (IGFBP1) was evaluated by real-time PCR in these animals.The results revealed that HOXA10 in the luteal phase primate endometrium is differentially expressed in the functionalis and the basalis zones, which is modulated in vivo by progesterone and also by the signals from the incoming embryo suggesting the involvement of $\mathrm{HOXA10}$ in the process of establishment of pregnancy in primates. In addition, the results also demonstrated that the expression of IGFBP1 but not HOXA11 is coregulated with HOXA10 in the endometria of these animals. The pattern of changes in the expression of HOXA10 in response to the two stimuli suggests that endometrial receptivity and implantation not only requires a synchrony of maternal and embryonic signaling on endometrial cells in the primates but there also exists a controlled differential response among the cells of various uterine compartments.
\end{abstract}

Reproduction (2007) 134 513-523

\section{Introduction}

Embryo implantation is a crucial step in mammalian reproduction that involves an intricate succession of genetic and cellular interactions, all of which must be synchronized and executed within an optimal time frame. The crucial events that occur in the maternal compartment that culminate in a successful pregnancy are (a) the achievement of receptive state in the endometrium and (b) the transformation of the stromal cells into the decidual cells. The ovarian steroid progesterone is an essential hormone that makes the uterus conducive ('receptive') to accept a blastocyst for implantation and subsequent decidualization (Wang \& Dey 2006). Several progesterone-regulated genes important for receptivity are being identified in the murine models (Bagchi et al. 2003, Wang \& Dey 2006) and a number of these have been shown to play essential roles in endowing receptivity in humans (Bagchi et al. 2003, Wang \& Dey 2006).
Beyond progesterone, recent studies have also suggested that the receptive stage endometrium is further primed by paracrine factors secreted by blastocyst prior to implantation. Studies in nonhuman primate model systems have demonstrated that extensive morphological remodeling and altered expression of cytokines and growth factors have been observed in the receptive stage endometrium of bonnet monkeys that were mated and the presence of an embryo had been verified (Rosario et al. 2005a, 2005b). Intriguingly, infusion of human chorionic gonadotropin and/or interleukin- $1 \beta$ at concentrations secreted by the blastocyst in the baboon endometrium also induces morphological changes in the endometrium and the expression of receptivityrelated genes is also altered (Fazleabas et al. 1999, Strakova et al. 2005). These observations lead to the speculation that along with progesterone, embryonic factors modulate the primate endometrium to endow receptivity for implantation and further maintenance of pregnancy. 
Transcription factors are elements that selectively bind to the appropriate DNA sequences on the promoter of the genes and regulate their expression and coordinate the process of cell differentiation. Since endometrial receptivity and decidualization are critical transformation processes for pregnancy to occur, it is likely that transcription factors whose expression in the endometrium is under the regulation of progesterone and embryonic stimuli would be critical for implantation (Zhao et al. 2006). Identification of such transcription factors would facilitate in expanding our knowledge of endometrial receptivity and initiation of pregnancy. One such transcription factor that is reported to play an important role in endometrial physiology is the homeobox A10 protein (HOXA10). HOXA10 is expressed in murine endometrium in a cycle-dependent manner and is regulated by progesterone (Satokata et al. 1995, Ma et al. 1999). Furthermore, the expression of HOXA10 is increased at the time of implantation and is maximal during decidualization, suggesting its role in initiation of pregnancy in the mouse (Satokata et al. 1995). Indeed, HOXA10 knockout mice are infertile owing to failure or abnormal implantation and inhibition of stromal cell decidualization (Benson et al. 1996, Lim et al. 1999). These observations underscore the importance of HOXA10 in the regulation of murine pregnancy. However, there exist fundamental differences in the mechanisms of implantation between the mouse and man, making it imperative to study the factors of interest in the primate system. But in the context of HOXA10, limited information exists on its roles in the regulation of implantation and pregnancy in humans. Expressed in a menstrual cycle-dependent manner in the human endometrium (Taylor et al. 1998, Gui et al. 1999), HOXA10 is downregulated in the endometria of women suffering from endometriosis (Browne \& Taylor 2006), polycystic ovary syndrome (Cermik et al. 2003) and idiopathic infertility (Gui et al. 1999). In vitro, HOXA10 expression is reportedly altered by steroids, embryo culture fluid, and hydrosalphinges (Sakkas et al. 2003, Daftary \& Taylor 2006). However, its in vivo regulation in the primate endometrium, particularly by progesterone and embryonic factors, is hitherto unknown. We hypothesized that if HOXA10 has a role in endometrial receptivity its expression must be modulated by progesterone and embryonic signals in the luteal phase endometrium around the time of implantation.

To gain more insights into the role of HOXA10 in primate uterus, particularly in the process of implantation and initiation of pregnancy, we decided to investigate, in detail, the expression profiles and in vivo regulation of both HOXA10 mRNA and protein by progesterone and embryonic stimuli during the window of receptivity in primates. As it is difficult to obtain human samples at these stages of pregnancy because of ethical limitations, we chose to study these events in bonnet monkeys. The reproductive physiology of bonnet monkeys is demonstrated to be closely similar to humans, especially in the context of menstrual cycle length, endometrial receptivity, and implantation (Katkam et al. 1995). In addition, the high fecundity rates of bonnet monkeys and the ready availability of an assay (preimplantation factor (PIF) bioassay) to detect preimplantation conceptus encouraged us to select this species for the present study.

HOXA10, under the influence of progesterone, has been shown to regulate the expression of a subset of progesterone-responsive genes. Among these is the HOXA10 ortholog HOXA11 and insulin-like growth factor-binding protein 1 (IGFBP1), which in murine models and in vitro have been proposed to be regulated by HOXA10 (reviewed in Daftary \& Taylor 2006). Hence, along with HOXA10, we also investigated the expression profiles of HOXA11 and IGFBP1 mRNA in the endometria of these animals.

\section{Materials and Methods}

The Institutional Animal Ethics committee and the Committee for the Purpose of Control and Supervision of Experiments on Animals, Ministry of Social Justice and Empowerment, Government of India approved the use of the animals for the study.

\section{Animals}

Adult bonnet monkeys (Macaca radiata) were housed singly under controlled conditions at the experimental animal facility of the institute and were fed with a diet composed of semi-formulated Indian bread, fresh seasonal fruits, eggs, and sterile water. Animals weighing $3.5-4.5 \mathrm{~kg}$, which had at least two consecutive ovulatory menstrual cycles of 28-30 days and normal steroid levels (peak estradiol $50-75 \mathrm{pg} / \mathrm{ml}$ and progesterone $300-600 \mathrm{pg} / \mathrm{ml}$ ), were used for the present study. The cyclicity was monitored and serum estradiol and progesterone concentrations were measured by specific RIA as described previously (Katkam et al. 1995, Sachdeva et al. 2001).

\section{Antiprogestin treatment}

To study the effects of progesterone, bonnet monkeys were treated with antiprogestin onapristone (ZK98.299) at a dose which is known to block endometrial receptivity without affecting the hypothalamus-pituitary-ovarian axis (Katkam et al. 1995). Our previous studies have demonstrated altered expression of a number of progesterone-regulated genes in the endometria of these animals (Puri et al. 2000, Sachdeva et al. 2001) indicating the validity of the model to study progesterone-mediated events in primate endometrium. ZK98.299 (a kind gift from Dr Walter Elger, Schering, 
Germany), at a dose of $5.0 \mathrm{mg}$ dissolved in vehicle (benzyl benzoate:castor oil, 9:1), was injected subcutaneously on day 1 of the menstrual cycle and continued every 3 days until the day of biopsy.

\section{Mated animals}

To investigate the regulation of HOXA10 expression by embryonic signals in vivo, endometria were collected from bonnet monkeys that were mated and the presence of embryo verified by a PIF bioassay (Rosario et al. 2005a). The hormone profiles, morphological changes, and the expression of some cytokines and growth factors in the endometria of these animals have been reported previously (Rosario et al. 2005a, 2005b). Bonnet monkeys show close similarity to humans in terms of menstrual cycle length, endometrial receptivity, and implantation (Katkam et al. 1995, Beier \& BeierHellwig 1998, Sachdeva et al. 2001, Lessey 2003, Rosario et al. 2005a). Implantation in this species is initiated approximately on days 6-7 post-ovulation (Jayaprakash et al. 1997).

Regularly cycling animals showing normal hormonal profiles were mated with males of proven fertility for six consecutive days starting from 2 days prior to the expected estradiol peak. PIF bioassay was performed as described previously (Rosario et al. 2005a) on a single serum sample collected on day 6 post-estradiol peak to detect the presence of preimplantation embryo in the mated bonnet monkeys. Endometria from PIF-positive mated animals were designated as those collected in conception cycles.

\section{Collection of endometrial biopsies}

Endometrial biopsies were collected on day 8 postestradiol peak from luteal controls $(n=5)$, antiprogestin treated $(n=3)$, and in conception cycles $(n=3)$ as described previously (Katkam et al. 1995). The day of biopsy coincides with the window of receptivity in these animals. A part of tissue was processed for routine paraffin embedding and sectioning. A piece of the tissue was freed from myometrium and flash frozen for RNA extraction.

\section{Isolation of RNA and real-time PCR}

Total RNA was extracted using the Trizol reagent (Invitrogen), treated with DNase (GE Healthcare, Hongkong, China), and reverse transcribed by random hexamer primers using Moloney murine leukemia virus as reverse transcriptase (Clontech) as described previously (Modi et al. 2005).
Specific primers were designed to amplify regions of human HOXA10, HOXA11, IGFBP1 and $18 S$ rRNA. The sequences for the same are given in Table 1.

\section{Real-time PCR}

The relative levels of HOXA10, HOXA11, and IGFBP1 mRNA in relation to $18 S$ rRNA (housekeeping) were estimated by iCycler real-time PCR system (Bio-Rad) using SYBR Green chemistry (Qiagen). For each primer pair, reaction efficiency was estimated by the amplification of serial dilution of human endometrial cDNA pool over a tenfold range.

The amplification conditions for HOXA10 were: initial denaturation at $95^{\circ} \mathrm{C}$ for 2 min followed by 40 cycles of denaturation at $94{ }^{\circ} \mathrm{C}$ for $30 \mathrm{~s}$, primer annealing at $65^{\circ} \mathrm{C}$ for $30 \mathrm{~s}$, and extension at $72^{\circ} \mathrm{C}$ for $1 \mathrm{~min}$. The final extension was carried out for $5 \mathrm{~min}$ at $72^{\circ} \mathrm{C}$. For HOXA11, IGFBP1, and 18S rRNA, amplifications were performed by specific primers (Table 1) and the conditions were the same as above, except that the annealing temperature was optimized at $60{ }^{\circ} \mathrm{C}$. The fluorescence emitted at each cycle was collected for the entire period of $30 \mathrm{~s}$ during the extension step of each cycle.

The homogeneity of the PCR amplicons was verified by running the products on $2 \%$ agarose gels and also by the melt curve method. All PCR amplifications were carried out in duplicate, and each experiment was repeated thrice to test the reproducibility. Mean $\mathrm{Ct}$ values generated in each experiment using the iCycler software (Bio-Rad) were used to obtain the standard curve, and the cDNA concentrations in the samples were computed and normalized to the housekeeping gene (18S RNA). The relative expression ratios were calculated manually using Pfaffl method (Pfaffl 2001) and statistically analyzed using one-way ANOVA.

\section{Immunohistochemistry for HOXA10 protein}

Five micrometers thick paraffin sections were cut and mounted on poly-L-lysine-coated slides. Immunohistochemistry was performed as described previously (Modi et al. 2003). Briefly, sections were deparaffinized in xylene, hydrated in descending grades of alcohol, and rinsed with $0.01 \mathrm{M} \mathrm{PBS}(\mathrm{pH}$ 7.2). After blocking in $1 \%$ donkey serum for $1 \mathrm{~h}$, the sections were probed overnight with an optimized concentration (1:30) of the primary goat polyclonal HOXA10 antibody (Santa Cruz Biotechnology, Santa Cruz, CA, USA). Negative controls were incubated with normal goat serum instead of the primary antibody. Human testicular sections collected previously (Modi et al. 2005) were used as tissue negative control. The next day the slides were washed five times with PBS and incubated with donkey anti-goat biotinylated secondary antibody (Santa Cruz Biotechnology) at a dilution of 1:200 for $2 \mathrm{~h}$. After five 
Table 1 Sequences of the primers used for real-time RT-PCR, the annealing temperatures, and the expected product size in base pairs.

\begin{tabular}{|c|c|c|c|}
\hline Gene & Sequence & Annealing temperature $\left({ }^{\circ} \mathrm{C}\right)$ & Product size (bp) \\
\hline HOXA10 & $\begin{array}{l}\text { F: } 5^{\prime} \text { GCCCCTTCCGAGAGCAGAAAAG 3' } \\
\text { R: } 5^{\prime} \text { AGGTGGAGCCTGCGGCTAATCTCTA 3' }\end{array}$ & 65 & 211 \\
\hline HOXA11 & $\begin{array}{l}\text { F: } 5^{\prime} \text { GATTTCTCCAGCCTCCCTTC } 3^{\prime} \\
\text { R: 5' AGTAGCAGTGGGCCAGATTG } 3^{\prime \prime}\end{array}$ & 60 & 175 \\
\hline IGFBP1 & $\begin{array}{l}\text { F: 5' GAGTTTAGCCAAGGCACAGG } 3^{\prime} \\
\text { R: } 5^{\prime} \text { GAGACCCAGGGATCCTCTTC } 3^{\prime}\end{array}$ & 60 & 173 \\
\hline $18 S$ & $\begin{array}{l}\mathrm{F}: 5^{\prime} \text { GGAGAGGGAGCCTGAGAAAC } 3^{\prime} \\
\text { R: } 5^{\prime} \text { CCTCCAATGGATCCTCGTTA } 3^{\prime}\end{array}$ & 60 & 171 \\
\hline
\end{tabular}

washes for $5 \mathrm{~min}$ each, the sections were incubated for $30 \mathrm{~min}$ with streptavidin-HRP (Santa Cruz Biotechnology) and then washed again in PBS. Detection was done using hydrogen peroxide (Qualigens, Mumbai, India) as substrate and diaminobenzidine (SigmaAldrich) as chromogen. All sections were briefly counterstained by Delafield's hematoxylin (Qualigens) and mounted in DPX. Slides were viewed under bright field using an upright microscope (Nikon 90I, Japan), and representative areas were photographed using a CCD camera and processed using an Image analysis software (ImagePro Plus 3.5, Media Cybernetics Inc., Bethesda, MD, USA).

Since the expression of HOXA10 was found to be heterogeneous and differences were found in the intensity of staining in the nuclei and cytoplasm, all the slides were manually scored. Three random sections from each animal of the three groups were scored for the intensity of staining (Table 2). $\mathrm{H}$-scores were calculated as $\mathrm{H}-$ score $=\sum(1+i) \mathrm{pi}$, where $i$ is the intensity score and $\mathrm{pi}$ is the percentage of cells showing that intensity (Cheon et al. 2001).

\section{Results}

\section{Cellular localization of HOXA10 in luteal phase endometria}

Figure 1 demonstrates the cellular localization of HOXA10 protein in the luteal phase endometria collected from bonnet monkeys during the window of receptivity. In all the endometrial sections investigated, HOXA10 expression was detected as brown staining in the nuclei and/or the cytoplasm of the luminal epithelium (LE), the glandular epithelium, and the stroma. Strong immunoreactivity for HOXA10 was observed in the cytoplasm and nuclei of the cells of the LE. However, along with nuclear expression, immunoreactive HOXA10 was detectable in the apical region of the luminal projections (Fig. 1B).

Immunoreactive HOXA10 was also detectable in the cells of the glandular epithelium, stroma, and blood vessels (Fig. 1C-E). However, the staining was heterogeneous with relatively stronger expression in the functionalis region (Fig. 1C) (defined as the upper twothird segment extending from the LE toward the myometrium) than the basalis (Fig. 1D, region just above the myometrium).

Semiquantative analysis by $\mathrm{H}$-scoring demonstrated an approximately twofold higher expression of HOXA10 in the functionalis when compared with the basalis region (Fig. 3A).

\section{HOXA10 expression in response to antiprogestin treatment}

To study whether progesterone regulates expression of endometrial HOXA10, the immunohistochemical localization of HOXA10 was compared in the luteal phase endometria of monkeys treated with the onapristone with those collected from untreated animals (controls) in the same phase.

When compared with luteal controls, in the antiprogestin-treated animals, the expression of HOXA10 was downregulated in the $L E$, stroma, and glands of the functionalis zone (Fig. 2B, F and J). When compared with controls, in antiprogestin-treated animals, the glandular epithelia of functionalis region had minimal expression of HOXA10; the stromal expression was also reduced (Fig. 2B and J).

In the LE, HOXA10 expression was eightfold lower when compared with controls, almost fourfold reductions were observed in the glands and the stroma of the functionalis zone of the antiprogestin-treated animals when compared with controls (Fig. 3B and F).

In the basalis region, the expression of HOXA10 in the stromal cells, glandular cells, and cells of blood vessels appeared to be higher when compared with controls;

Table 2 Criteria for scoring the immunostaining of HOXA10 in the monkey endometrium.

\begin{tabular}{ll}
\hline Score & Intensity \\
\hline 0 & No staining \\
1 & Weak cytoplasmic \\
2 & Moderate cytoplasmic \\
3 & Weak nuclear and cytoplasmic \\
4 & Strong cytoplasmic \\
5 & Strong nuclear \\
6 & Moderate cytoplasmic and nuclear \\
7 & Strong cytoplasmic and nuclear \\
\hline
\end{tabular}



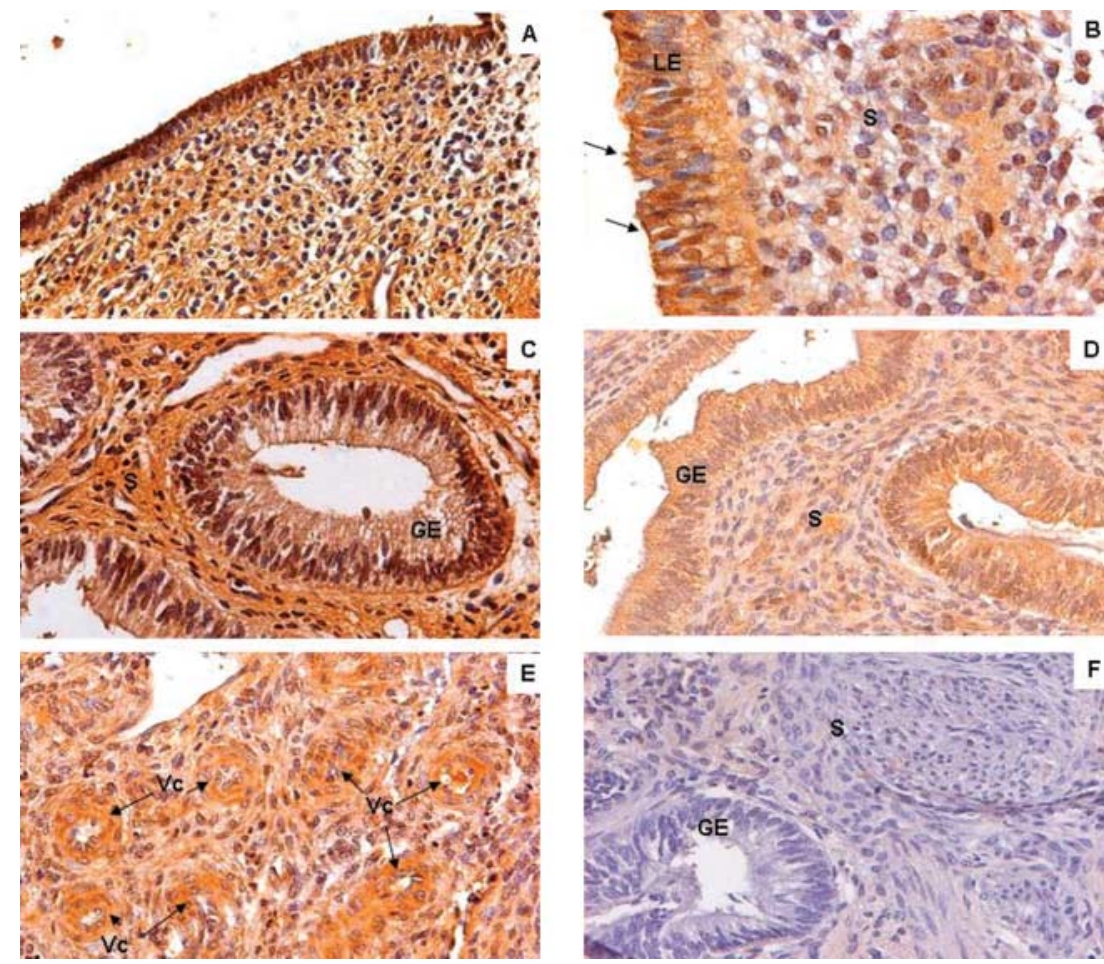

Figure 1 Immunohistochemical localization of HOXA10 in the luteal phase bonnet monkey endometrium. (A and B) Luminal epithelium (LE); note the strong staining in the apical projections (arrow in B). (C) Functionalis zone, (D) Basalis zone, (E) Blood vessels $\left(\mathrm{VC}_{\mathrm{C}}\right.$ ) in the functionalis (arrows). (F) Negative control. Magnification A $40 \times$, B $100 \times$, and digitized, C-F $60 \times$. however, this difference was not statistically significant (Figs $2 \mathrm{~F}$ and $\mathrm{N}$ and $3 \mathrm{~B}$ ).

\section{HOXA10 expression in the endometrium in response to embryonic stimuli}

To study whether HOXA10 expression is regulated by the signals from blastocyst, endometria from mated bonnet monkeys (where the presence of blastocyst was verified: conception cycles) were analyzed. Figure 2 gives the comparative profiles of HOXA10 immunoexpression in the luteal phase endometria from conception and nonconception cycles (control). The corresponding mean $\mathrm{H}$-scores are depicted in Fig. 3C. As evident, HOXA10 protein was barely detectable in the cells of the LE of endometria from conception cycle (Fig. 2 C), the mean $\mathrm{H}$-score was eightfold lower when compared with luteal controls. In contrast to the LE, in the endometria from conception cycles, intense staining for HOXA10 was observed in the nuclei and cytoplasm of the glandular, stromal, and vascular cells in the functionalis and basalis zone (Fig. 2C, G, K and O). The expression was higher when compared with respective luteal controls.

The mean $\mathrm{H}$-scores for HOXA10 staining in the endometria from conception cycles were also significantly different with an approximately twofold increase in the level of expression in the glands and stroma when compared with controls. The mean $\mathrm{H}$-score was also higher in the case of cells of the blood vessels analyzed in endometria from conception cycles when compared with controls (Fig. 3C).

\section{Relative expression of HOXA10 mRNA in endometria of antiprogestin-treated and mated bonnet monkeys}

To investigate whether the changes in protein expression of HOXA10 are also reflected at the transcript level, realtime PCR analysis was carried out. Single bands of expected size and sequences were observed after PCR amplification of endometrial cDNA with specific primers for HOXA10 and $18 S$ (not shown). The presence of single PCR products in real time was also verified by the melt curve analysis (not shown).

Figure 4A gives the mean relative expression of HOXA10 mRNA (normalized to 185 ) in the endometria of bonnet monkeys when compared with an internal reference cDNA. In comparison with controls, the transcript levels of HOXA10 were 20-fold lower in the endometria from antiprogestin-treated animals. Conversely, an almost tenfold increase in the transcript levels of HOXA10 was observed in the endometria from conception cycles when compared with luteal controls.

\section{Relative expression levels of IGFBP1 and HOXA11 in the endometria of control antiprogestin-treated and mated bonnet monkeys}

Single bands of expected size and sequence were observed after PCR amplification of endometrial cDNA 

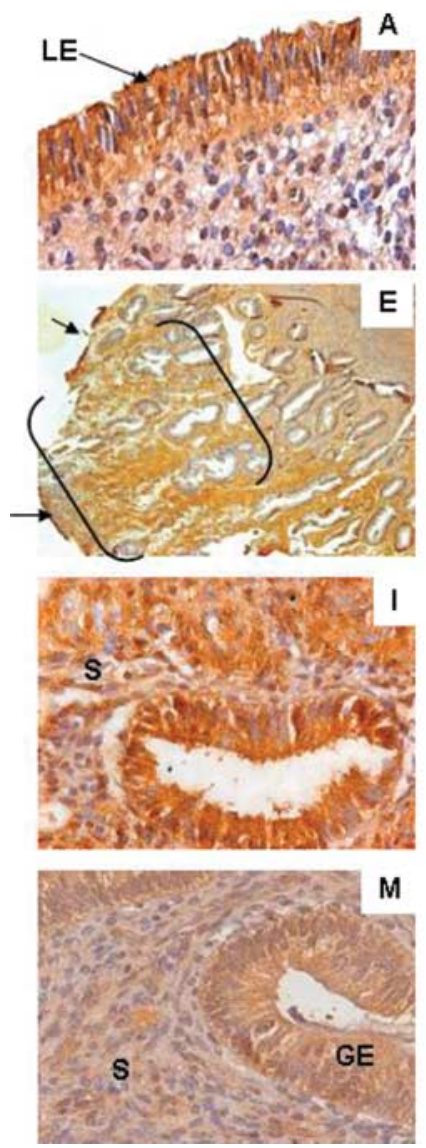
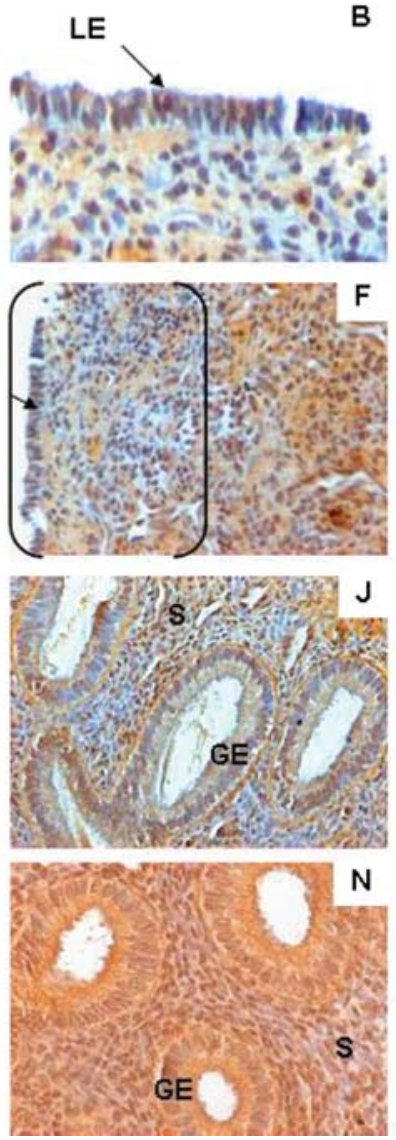
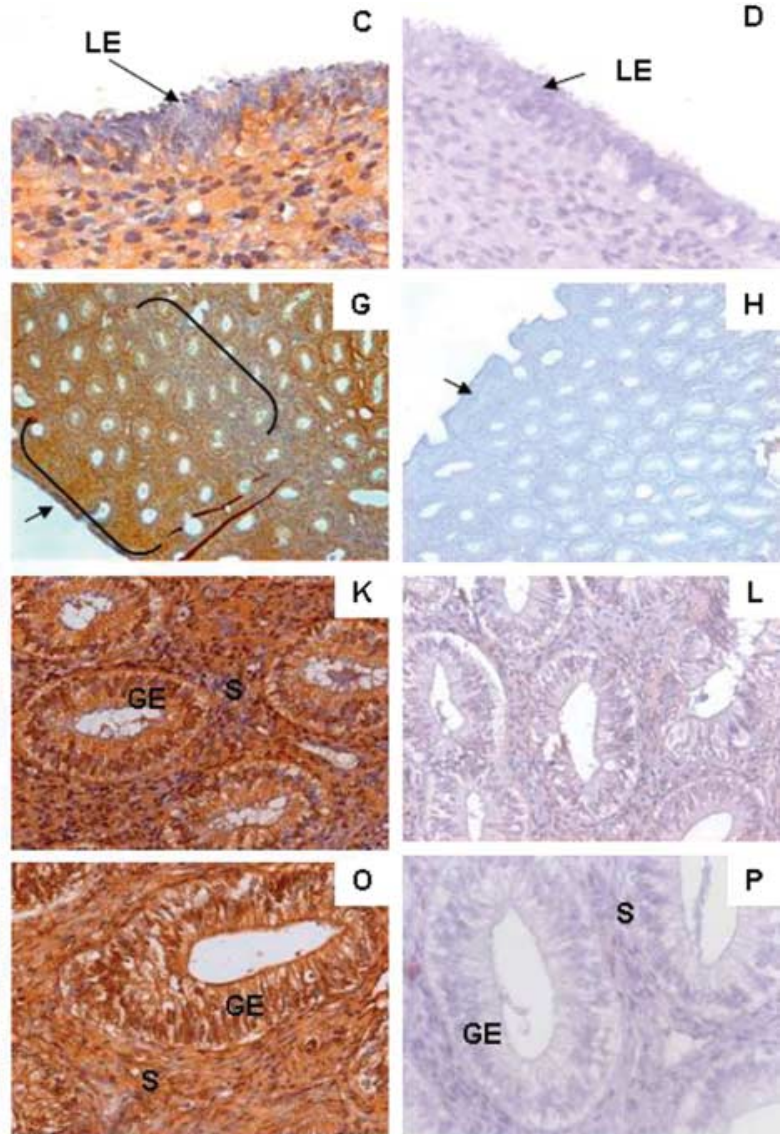

Figure 2 Effects of antiprogestin treatment and embryonic stimuli on the expression of HOXA10 in the endometrium. A-D luminal epithelium from controls (A), antiprogestin-treated (B), and conception cycle (C) animals. The corresponding negative control section is shown in D. E-H are low magnification images of the endometria of control (E), antiprogestin-treated (F), and conception cycle $(\mathrm{G})$ animals. The negative control section is shown in $\mathrm{H}$. The direction of luminal epithelium (LE) is shown by arrow and the functionalis region is represented in the brackets. Note the differential expression of HOXA10 in the control and antiprogestin-treated animals. I-L are representative photographs of the endometrium functionalis from control (I), antiprogestin-treated (J), and conception cycle (K) animals. Negative control is represented in (L). M-Pare representative photographs of the basalis from control (I), antiprogestin-treated $(\mathrm{J})$, and conception cycle $(\mathrm{K})$ animals. $\mathrm{P}$ is the negative control sections. Magnifications A, B, and C $60 \times$ and digitized, E-H 4X, I-P 40×, and D 20×. GE, glandular epithelium; S, stroma.

with specific primers for IGFBP1 and HOXA11. The presence of single PCR products in real time was also verified by the melt curve analysis (not shown). Figure 4B and $\mathrm{C}$ gives the mean relative expression of IGFBP1 and HOXA11 mRNA (normalized to 185 ) respectively in the endometria of bonnet monkeys when compared with an internal reference sample.

When compared with controls, IGFBP1 mRNA was significantly downregulated in the endometria of antiprogestin-treated animals. In the endometria of animals from conception cycles an almost fivefold increase in the transcript levels of IGFBP1 was observed when compared with luteal controls.

When compared with luteal controls, HOXA11 mRNA was significantly reduced in the endometria of antiprogestin-treated animals; the levels were identical to controls in the case of endometria of animals in conception cycles.

\section{Discussion}

The synchronous development of the endometrium for implantation is highly dependent on ovarian steroids, particularly progesterone; there is growing evidence that the embryo further modulates the endometrial milieu making it competent for implantation. In the present study, using nonhuman primate as models, we have shown that the homeobox protein HOXA10 in the luteal phase endometrium is differentially expressed in the functionalis and the basalis zones. This expression is modulated in vivo by progesterone and also by the signals from the incoming embryo, suggesting the involvement of HOXA10 in the process of establishment of pregnancy in primates. In addition, the results of the present study also demonstrate that the expression of IGFBP1 but not HOXA11 is coregulated with HOXA10.

The mammalian gene for HOXA10 is a member of the drosophila $A b d B$ class of homeodomain proteins that 


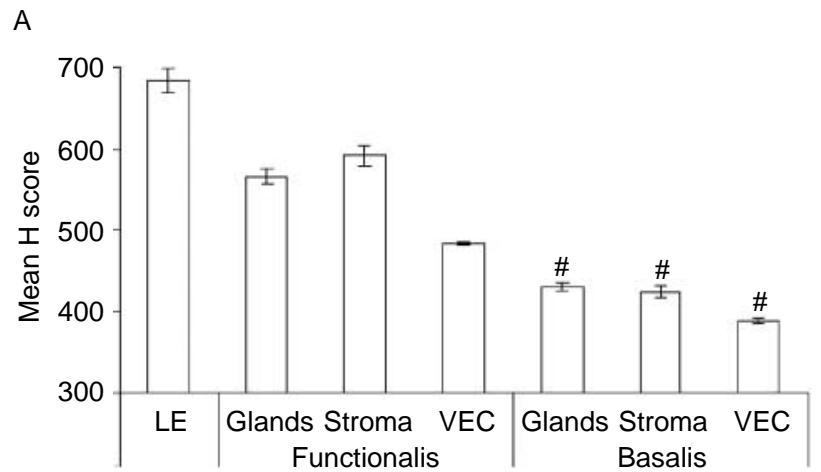

B

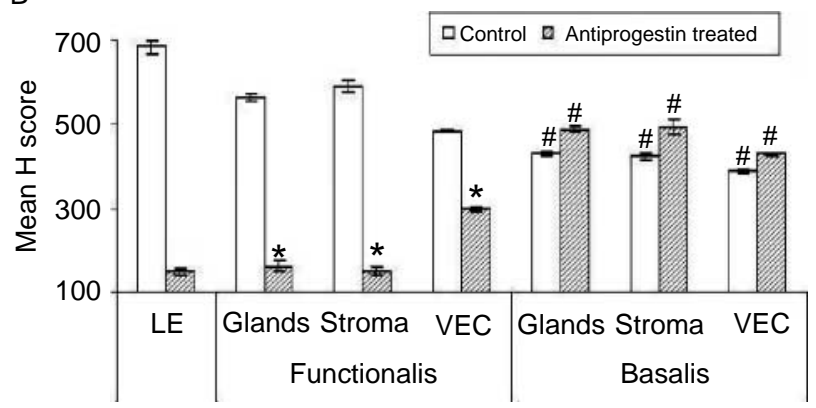

C

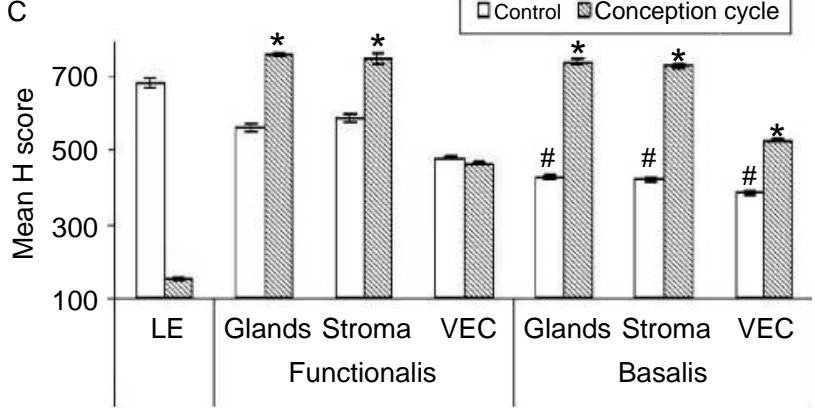

Figure 3 Semiquantitative analysis of HOXA10 immunostaining using the $\mathrm{H}$-score method (see text for details). (A) Comparison of the mean $\mathrm{H}$-scores of HOXA10 staining in the luminal epithelium (LE), glands, and stroma and vascular cells (VEC) from the functionalis and basalis. (B) Comparison of the expression of HOXA10 in various endometrial compartments (as in A) in control (open bars) and antiprogestin-treated (slashed bars) animals. (C) Comparison of the $\mathrm{H}$-scores in the various compartments (as in A) of endometria from control (open bars) and conception cycle (slashed bars) animals. All values are mean \pm s.E.M. * Statistically significant $(P<0.05)$ when compared with control,

${ }^{*}$ significantly different $(P<0.05)$ when compared with functionalis region.

play a crucial role in uterine homeosis (Daftary \& Taylor 2006). Expressed in developing mouse and human uterus (Daftary \& Taylor 2006), HOXA10 gene expression has also been detected in the adult endometrium (Satokata et al. 1995, Taylor et al. 1998, Ma et al. 1999, Daftary \& Taylor 2006). To gain insights into the roles of HOXA10 in the adult endometrium, particularly in the process of endometrial receptivity in the primates, we analyzed the spatial distribution of
HOXA10 protein in the endometrium during 'window of receptivity' in the endometria of bonnet monkeys. Corroborating the observations in human endometrium (Gui et al. 1999, Browne \& Taylor 2006), HOXA10 protein was immunolocalized in the nuclei and cytoplasm of the epithelial, stromal, and endothelial cells of the monkey endometrium with the most abundant expression in the luminal epithelial cells. While the presence of HOXA10 in the human endometrium has been reported, a unique observation made in this study was the difference in its spatial distribution within the luteal phase endometrium. In all the sections examined, a perceptible, intense staining for HOXA10 was evident in the functionalis region below the LE but the staining was conspicuously lower in the basalis region extending toward the myometrium. This observation indicates a possible differential requirement of HOXA10-regulated transcriptome in the two compartments. Such zone-specific, differential expression of some genes has been observed in the primate endometrium (Rudolph-Owen et al. 1998, Torres et al. 2002, Slayden \& Brenner 2004); these are thought to be involved in endowing specialized roles to the functionalis zone for implantation and also in the regulation of menstruation (Rudolph-Owen et al. 1998, Torres et al. 2002). Since the HOX family of genes is known to have a role in body segmentation by regulating the differential distribution of the morphogens (Pearson et al. 2005), it is tempting to hypothesize that the zone-specific distribution of HOXA10 would lead to creation of precincts of microenvironments within the uterus in preparation for implantation. It will be of interest to identify and study the zonal distribution of HOXA10regulated genes within the endometrium to evaluate this hypothesis.

An important feature of most implantation-related genes is their regulation by progesterone. Based on a number of experimental studies, it is clear that most genes playing a regulatory role in the process of endometrial receptivity or implantation are regulated by progesterone (Bagchi et al. 2003). To investigate whether HOXA10 is under the regulation of progesterone in primate endometrium, its expression was studied in the endometria of animals treated with onapristone. We have earlier shown that onapristone, at the specified doses in bonnet monkey, does not affect the hypothalamus-pituitary-ovarian axis but blocks implantation in the bonnets (Katkam et al. 1995) and the expression of some progesterone-regulated genes is also altered (Puri et al. 2000, Sachdeva et al. 2001, Patil et al. 2005). In the present study, we found that blocking progesterone actions (by onapristone treatment) lead to suppression of HOXA10 mRNA and protein expression in the endometrium. These results are in agreement with the observations where, in the mouse, administration of antiprogestin RU486 leads to suppression of Hoxa10 mRNA (Ma et al. 1999) and treatment of cultured human 

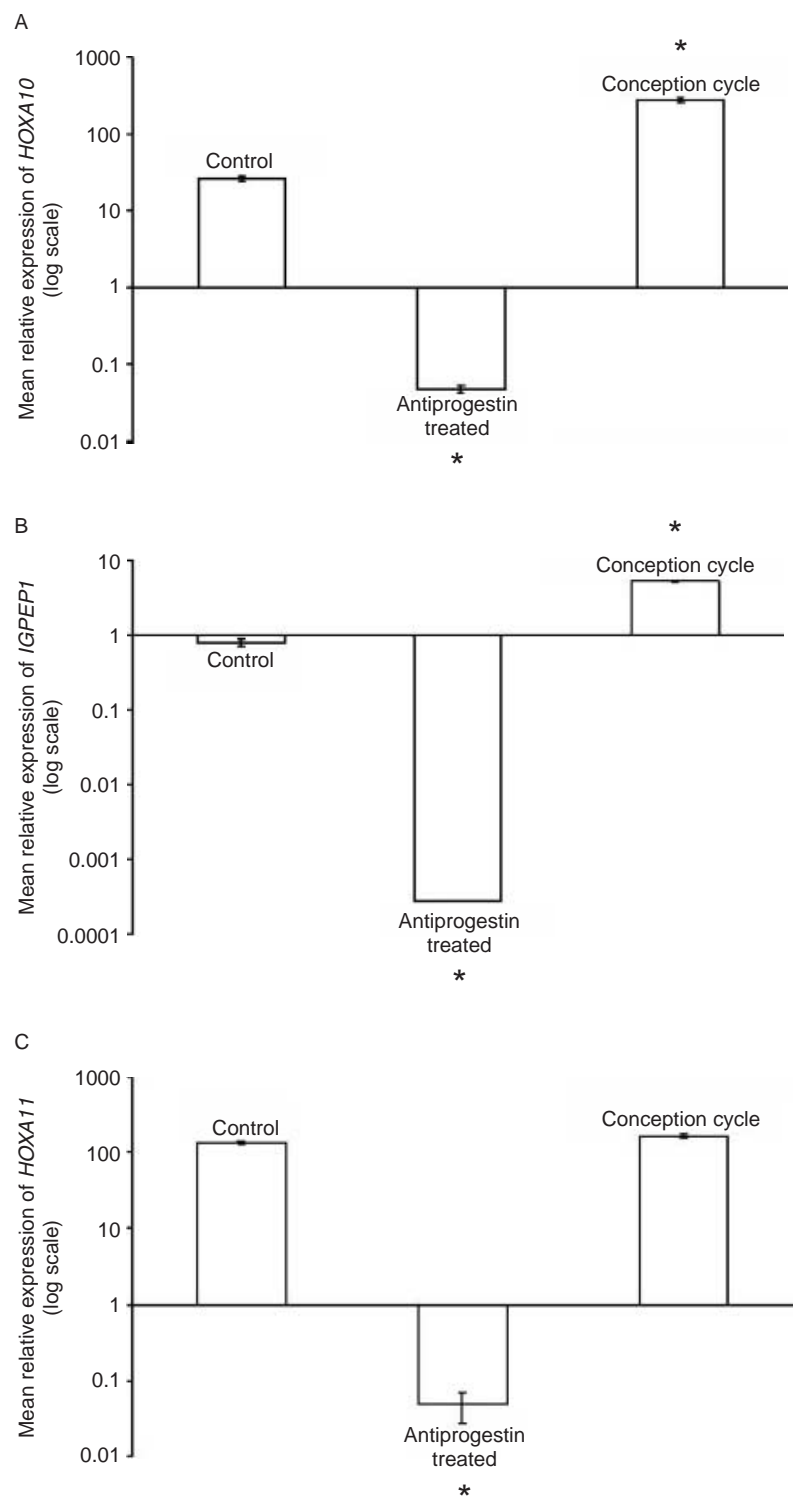

Figure 4 Expression of HOXA10 (A), IGFBP1 (B), and HOXA11 (C) mRNA in the endometria of control, antiprogestin-treated, and conception cycle animals. Values are mean \pm s.E.M. of relative expression normalized to $18 S$ RNA estimated by real-time PCR. *Statistically significant $(P<0.05)$ when compared with control.

endometrial epithelial or stromal cells with progesterone leads to increase in its expression (Taylor et al. 1998, Gui et al. 1999). These in vitro observations coupled with the in vivo data obtained herein provide strong evidence to support the regulatory role of progesterone in the endometrium. Interestingly, progesterone-responsive elements have been identified by in silico analysis of the murine (Ma et al. 1999) and human HOXA10 promoter regions (Modi, unpublished data).

Although the expression of HOXA10 was downregulated in endometria of monkeys treated with antiprogestin, the response of antiprogestin treatment in the two zones of the endometrium was different.
While HOXA10 expression was significantly lower in the functionalis zone, the expression in the basalis region was unaltered in the endometria of antiprogestin-treated animals when compared with the controls. These observations imply that HOXA10 expression is under the control of progesterone at least in the functionalis; the basalis region may not be so responsive to alterations in progesterone actions. Indeed, it is known that functionalis zone is more sensitive to progesterone actions when compared with basalis in the primates (Slayden \& Brenner 2004). In the basalis region, it is likely that HOXA10 expression may be maintained by mechanisms other than those mediated by progesterone. Interestingly, in vitro experiments in uterine cells have demonstrated an autoregulatory mechanism where HOXA10 acts on its own promoter and induces its transcription (Kelly et al. 2006). Whether such mechanisms exist in vivo to maintain the expression of HOXA10 in the basalis needs to be evaluated.

Having observed that HOXA10 is differentially expressed in the two zones of luteal phase endometrium and is modulated by progesterone, we next investigated the in vivo effects of embryonic signals on the cellular expression of HOXA10. Endometria of animals in the conception cycles (where the presence of an embryo had been verified) had tenfold higher mRNA levels of HOXA10 when compared with controls; the immunoreactive HOXA10 was also found to be higher. Abundant expression of HOXA10 protein was evident in the cytoplasm and nuclei of stromal and the glandular cells in the functionalis and basalis zones; the expression was higher in the stroma when compared with the glands. Thus, it appears that the stromal cells are more responsive than the epithelial cells to embryonic stimuli. Indeed, mice knockout for Hoxa10 have defective stromal cell proliferation and differentiation around the time of implantation (Benson et al. 1996, Lim et al. 1999), suggesting a dominant role of HOXA10 in the stromal cells.

Interestingly, studies on the cellular distribution of HOXA10 in the endometria in conception cycles revealed some unanticipated findings. An unexpected and surprising observation made in this study was the reduced expression of HOXA10 protein in the luminal epithelial cells in the endometria of animals in conception cycles when compared with controls. While the endometrial LE in the nonconception cycles abundantly expressed HOXA10, the protein was conspicuously absent in most cells including those forming pre-epithelial plaque (not shown). This observation was surprising, as in vivo studies in the mouse had shown that suppression of HOXA10, particularly in the epithelial cells, leads to inhibition of embryo implantation; forced overexpression leads to increase in the litter size (Bagot et al. 2000). Additionally, spent IVF medium (where embryos had been cultured) stimulates HOXA10 expression in the epithelial cells in vitro 
(Sakkas et al. 2003). At present, it is difficult to explain the reason for such discrepant findings, but the present observations tempt us to hypothesize that the products of HOXA10-modulated transcriptome in the LE may be inhibitory for implantation and hence may be downregulated by embryonic stimuli.

Another intriguing observation made in this study is the differential response of embryonic stimuli on the expression of HOXA10 in the luminal and glandularepithelium. While HOXA10 expression was reduced in LE of the conception cycles when compared with nonconception cycles, the expression in glandular epithelium was elevated. A similar differential regulation of the glycoprotein MUC1 has been reported in the endometria of macaques during early pregnancy (Julian et al. 2005). The selective reduction in the expression of important proteins like HOXA10 and MUC1 in the luminal but not glandular epithelia in conception cycles reflect the fundamental distinctions among epithelia of the two uterine compartments. It also indicates the existence of differential mechanisms of modulation of gene expression within these compartments in conception versus the nonconception cycles. However, what modulates such differential responses of the luminal versus the glandular epithelia in the endometria of conception cycles is difficult to speculate.

Another striking observation made in the endometria of animals in conception cycle is the complete loss of differential expression of HOXA10 in the functionalis versus basalis zones. While HOXA10 was abundantly expressed in the functionalis when compared with basalis zone in nonconception cycle, it was equally and more abundantly expressed in both the zones of endometria in the conception cycles. This suggests that the zonal restrictions observed in the nonconception cycles are lost in the presence of embryo and the involvement of both the zones is required for implantation.

The alterations in the expression of HOXA10 in response to progesterone and embryonic stimuli prompted us to evaluate the expression profiles of HOXA10-regulated genes in both these models. For this purpose, we chose to analyze the expression profiles of IGFBP1 and HOXA11 as both these factors not only share close physical proximity with HOXA10 in the human genome but are also known to be regulated by HOXA10 (Kim et al. 2003, Yao et al. 2003). In the endometria of antiprogestin-treated animals, expression of IGFBP1 was significantly lower when compared with controls; in the mated animals, IGFBP1 mRNA levels were more than fivefold higher. These observations confirm our previous findings that decidualization is initiated in the endometria of animals in the conception cycles prior to implantation (Rosario et al. 2005a) and further suggest that IGFBP1 expression is coregulated with HOXA10. Intriguingly, HOXA10-binding sites have been identified in the IGFBP1 promoter and recombinant HOXA10 activates IGFBP1 promoter in vitro in the endometrial stromal cells (Kim et al. 2003). These observations together suggest that the coordinated expression of HOXA10 is essential to maintain IGFBP1 activity in the endometrial cells during implantation.

Akin to HOXA10 and IGFBP1, in the antiprogestintreated animals the expression of HOXA11 mRNA was downregulated when compared with controls suggesting that the expression of HOXA11 may be directly or indirectly regulated by progesterone via HOXA10. Indeed, HOXA11 is induced by progesterone treatment in the uterus (Yao et al. 2003, Daftary \& Taylor 2006); HOXA11 mRNA expression is markedly attenuated in the uteri of progesterone-treated HOXA10 mutant mice (Yao et al. 2003), indicating that the expression of HOXA10 and 11 may be coregulated. However, this phenomenon appears to be restricted to the progesterone effects, as in the endometria from conception cycles where HOXA10 expression was higher, the expression of HOXA11 was identical to controls. This observation indicates that HOXA11 transcription in the endometrium is not affected by embryonic stimuli and the expression of both these orthologs may not be strictly coregulated during the process of implantation. Indeed, no correlation has been found in expression profiles of HOX orthologs in human endometrium during different phases of menstrual cycle (Akbas \& Taylor 2004), indicating a lack of redundancy and existence of independent regulatory mechanisms.

To summarize, using bonnet monkey as a study model, the results of the present study demonstrate that HOXA10 is differentially expressed in the functionalis and basalis regions of the luteal phase endometrium and its expression is modulated by progesterone and embryonic stimuli in vivo. The pattern of changes in the expression of HOXA10 in response to the two stimuli suggests that endometrial receptivity and implantation not only requires a synchrony of maternal and embryonic signaling on endometrial cells but also controlled differential response among the cells of various uterine compartments. Identification of genes regulated by HOXA10 in the different cell types of the primate endometrium would help us understand local control of endometrial receptivity and implantation. This information is envisaged to have important implication in the development of endometrium-based anti-implantation drugs for contraception, developing protocols for modulating the length of window of receptivity to maximize pregnancy rates in assisted reproductive technology programs, and also in diagnosis and treatment of female infertility.

\section{Acknowledgements}

We express our gratitude to Dr Vishakha Patil, Dr Gracy Rosario, Dr R Katkam and Dr D Manjaramkar for their help in the collection of monkey tissues. This work (NIRRH/MS/19/2006) was funded by grants from the 
Department of Biotechnology, Ministry of Science and Technology, Government of India and Indian Council of Medical Research, Ministry of Health and Family Welfare, Government of India. We are grateful to the Lady Tata Memorial Trust (Mumbai, India) for the JRF to GBG. The data presented here are part of the studies toward doctoral studies by GBG. The authors declare that there is no conflict of interest that would prejudice the impartiality of this scientific work.

\section{References}

Akbas GE \& Taylor HS 2004 HOXC and HOXD gene expression in human endometrium: lack of redundancy with HOXA paralogs. Biology of Reproduction 70 39-45.

Bagchi IC, Cheon YP, Li Q \& Bagchi MK 2003 Progesterone receptorregulated gene networks in implantation. Frontiers in Bioscience $\mathbf{8}$ s852-s861.

Bagot CN, Troy PJ \& Taylor HS 2000 Alteration of maternal Hoxa10 expression by in vivo gene transfection affects implantation. Gene Therapy 7 1378-1384.

Beier HM \& Beier-Hellwig K 1998 Molecular and cellular aspects of endometrial receptivity. Human Reproduction Update 4 448-458.

Benson GV, Lim H, Paria BC, Satokata I, Dey SK \& Mass RL 1996 Mechanisms of reduced fertility in Hoxa-10 mutant mice: uterine homeosis and loss of maternal Hoxa-10 expression. Development 122 2687-2696.

Browne H \& Taylor H 2006 HOXA10 expression in ectopic endometrial tissue. Fertility and Sterility 85 1386-1390.

Cermik D, Selam B \& Taylor HS 2003 Regulation of HOXA-10 expression by testosterone in vitro and in the endometrium of patients with polycystic ovary syndrome. Journal of Clinical Endocrinology and Metabolism 88 238-243.

Cheon KW, Lee H, Parhar IS \& Kang IS 2001 Expression of the second isoform of gonadotrophin-releasing hormone $(\mathrm{GnRH}-\mathrm{II})$ in human endometrium throughout the menstrual cycle. Molecular Human Reproduction 7 447-452.

Daftary GS \& Taylor HS 2006 Endocrine regulation of HOX genes. Endocrine Reviews 27 331-355.

Fazleabas AT, Donnelly KM, Srinivasan S, Fortman JD \& Miller JB 1999 Modulation of the baboon (Papio anubis) uterine endometrium by chorionic gonadotrophin during the period of uterine receptivity. PNAS 96 2543-2548.

Ghosh D \& Sengupta J 1988 Patterns of estrogen and progesterone receptors in rhesus monkey endometrium during secretory phase of normal menstrual cycle and preimplantation stages of gestation. Journal of Steroid Biochemistry 31 223-229.

Gui Y, Zhang J, Yuan L \& Lessey BA 1999 Regulation of HOXA-10 and its expression in normal and abnormal endometrium. Molecular Human Reproduction 5 866-873.

Jayaprakash D, Satish KS, Ramachandra SG, Ramesh V \& Seshagiri PB 1997 Successful recovery of preimplantation embryos by nonsurgical uterine flushings in the bonnet monkeys. Theriogenology 47 1019-1026.

Julian J, Enders AC, Fazleabas AT \& Carson DD 2005 Compartmental distinctions in uterine Muc-1 expression during early pregnancy in cynomolgous macaque (Macaca fascicularis) and baboon (Papio anubis). Human Reproduction 20 1493-1503.

Katkam RR, Gopalkrishnan K, Chwalisz K, Schillinger E \& Puri CP 1995 Onapristone (ZK 98.299): a potential antiprogestin for endometrial contraception. American Journal of Obstetrics and Gynecology 173 779-787.

Kelly M, Daftary G \& Taylor HS 2006 An autoregulatory element maintains HOXA10 expression in endometrial epithelial cells. American Journal of Obstetrics and Gynecology 194 1100-1107.
Kim JJ, Taylor HS, Akbas GE, Foucher I, Trembleau A, Jaffe RC, Fazleabas AT \& Unterman TG 2003 Regulation of insulin-like growth factor binding protein-1 promoter activity by FKHR and HOXA10 in primate endometrial cells. Biology of Reproduction 68 24-30.

Lessey BA 2003 Two pathways of progesterone action in the human endometrium: implications for implantation and contraception. Steroids 68 809-815.

Lim H, Ma L, Ma WG, Maas RL \& Dey SK 1999 Hoxa-10 regulates uterine stromal cell responsiveness to progesterone during implantation and decidualization in the mouse. Molecular Endocrinology 13 1005-1017.

Ma L, Benson G. Lim, Dey S \& Maas R 1999 The role of abdominal B Hoxa genes during implantation. In Embryo Implantation: Molecular Cellular and Clinical Aspects, pp 249-260. Ed. D Carson. USA; New York: Serono Symposia; Springer-Verlag.

Modi D, Sane S \& Bhartiya D 2003 Accelerated germ cell apoptosis in sex chromosome aneuploid fetal human gonads. Molecular Human Reproduction 9 219-222.

Modi D, Shah CA, Sachdeva G, Gadkar S, Bhartiya D \& Puri CP 2005 Ontogeny and cellular localization of SRY transcripts in the human testes and its detection in spermatozoa. Reproduction 130 603-613.

Patil VS, Sachdeva G, Modi DN, Katkam RR, Manjramkar DD, Hinduja I \& Puri CP 2005 Rab coupling protein (RCP): a novel target of progesterone action in primate endometrium. Journal of Molecular Endocrinology 35 357-372.

Pearson JC, Lemons D \& McGinnis W 2005 Modulating Hox gene functions during animal body patterning. Nature Reviews. Genetics 6 893-904.

Pfaffl MW 2001 A new mathematical model for relative quantification in real-time RT-PCR. Nucleic Acids Research 29 2002-2007.

Puri CP, Katkam RR, Sachdeva G, Patil V, Manjramkar DD \& Kholkute SD 2000 Endometrial contraception: modulation of molecular determinants of uterine receptivity. Steroids $\mathbf{6 5}$ 783-794.

Rosario GX, Modi DN, Sachdeva G, Manjramkar DD \& Puri CP 2005a Morphological events in the primate endometrium in the presence of a preimplantation embryo, detected by the serum preimplantation factor bioassay. Human Reproduction 20 61-71.

Rosario GX, Sachdeva G, Manjramkar DD, Modi DN, Meherji PK \& Puri CP 2005b Endometrial expression of immunomodulatory cytokines and their regulators during early pregnancy in bonnet monkeys (Macaca radiata). Human Reproduction 20 3039-3046.

Rudolph-Owen LA, Slayden OD, Matrisian LM \& Brenner RM 1998 Matrix metalloproteinase expression in Macaca mulatta endometrium: evidence for zone-specific regulatory tissue gradients. Biology of Reproduction 59 1349-1359.

Sachdeva G, Patil V, Katkam RR, Manjramkar DD, Kholkute SD \& Puri CP 2001 Expression profiles of endometrial leukemia inhibitory factor, transforming growth factor beta2 (TGFbeta2), and TGFbeta2 receptor in infertile bonnet monkeys. Biology of Reproduction 65 1-8.

Sakkas D, Lu C, Zulfikaroglu E, Neuber E \& Taylor HS 2003 A soluble molecule secreted by human blastocysts modulates regulation of HOXA10 expression in an epithelial endometrial cell line. Fertility and Sterility $801169-1174$.

Satokata I, Benson G \& Maas R 1995 Sexually dimorphic sterility phenotypes in Hoxa10-deficient mice. Nature 374 460-463.

Slayden OD \& Brenner RM 2004 Hormonal regulation and localization of estrogen, progestin and androgen receptors in the endometrium of nonhuman primates: effects of progesterone receptor antagonists. Archives of Histology and Cytology 67 393-409.

Strakova Z, Mavrogianis P, Meng X, Hastings JM, Jackson KS, Cameo P, Brudney A, Knight O \& Fazleabas AT 2005 In vivo infusion of interleukin-1 beta and chorionic gonadotropin induces endometrial changes that mimic early pregnancy events in the baboon. Endocrinology 146 4097-4104.

Taylor HS, Arici A, Olive D \& Igarashi P 1998 HOXA10 is expressed in response to sex steroids at the time of implantation in the human endometrium. Journal of Clinical Investigation 101 1379-1384. 
Torres MS, Ace CI \& Okulicz WC 2002 Assessment and application of laser microdissection for analysis of gene expression in the rhesus monkey endometrium. Biology of Reproduction 67 1067-1072.

Wang H \& Dey S 2006 Roadmap to embryo implantation clues from mouse models. Nature Reviews. Genetics 7 185-199.

Yao MW, Lim H, Shust D, Choe S, Farago A, Ding Y, Michaud S, Church G \& Maas R 2003 Gene expression profiling reveals progesteronemediated cell cycle and immunoregulatory roles of Hoxa-10 in the preimplantation uterus. Molecular Endocrinology 17 610-627.
Zhao B, Koon D \& Bethin KE 2006 Identification of transcription factors at the site of implantation in the later stages of murine pregnancy. Reproduction 131 561-571.

Received 21 May 2007

First decision 15 June 2007

Accepted 15 June 2007 